\title{
Computation of Hermite Polynomials
}

\author{
By Laurance C. Eisenhart* and George E. Trapp, Jr.
}

\begin{abstract}
Projection methods are commonly used to approximate solutions of ordinary and partial differential equations. A basis of the subspace under consideration is needed to apply the projection method. This paper discusses methods of obtaining a basis for piecewise polynomial Hermite subspaces. A simple recursive procedure is derived for generating piecewise Hermite polynomials. These polynomials are then used to obtain approximate solutions of differential equations.
\end{abstract}

1. Introduction. Projection methods using piecewise Hermite polynomials have been applied to the approximate solution of differential equations. A partial list of contributors would include: Birkhoff, Curry, Goel, Jerome, Lanczos, Schoenberg, Schultz and Varga [1], [3], [5], [7], [9], [10], [12], [14]. In particular, Varga and Jerome ([7] and [14]) discuss the use of projection methods for ordinary differential equations. Piecewise polynomial subspaces are useful because the solution matrices are well suited for inversion procedures.

The above authors have shown the advantages of higher order polynomials in increased accuracy. In this paper, we present a method of constructing these higher order polynomials. Goel in [5] has considered this problem. His results are primarily for low order polynomials.

We begin by showing that the construction of piecewise Hermite polynomials of degree $2 M-1$ is equivalent to inverting an $M$ by $M$ ill-conditioned matrix. Because of the ill-conditioning, we develop a recursive procedure to generate the polynomials.

We construct the polynomials on the interval $[0,2]$, then we note that a simple change of variable gives the required polynomials for any mesh structure (see [12]).

Finally, we illustrate the use of these polynomials by obtaining numerical approximations for ordinary differential equations.

2. Statement of the Problem. Given an ordinary differential equation on an interval $[a, b]$, a mesh structure is defined by

$$
a=x_{0}<x_{1}<\cdots<x_{N}<x_{N+1}=b .
$$

An approximate solution is sought in a finite-dimensional Hermite subspace of the appropriate Sobolev space, where a weak solution is known to exist [16]. The piece-

Received October 6, 1971.

AMS (MOS) subject classifications (1970). Primary 65D20; Secondary 65L99, 65Q05.

Key words and phrases. Hermite polynomials, piecewise polynomials, projection method.

* The work was done while the first author was at Westinghouse Electric Corporation, Penn Center Site, Pittsburgh, Pennsylvania 15235. 
wise polynomials, which are used in the projection from the Sobolev space to the finite-dimensional Hermite space, are required to have nonzero support only in an interval of the form $\left[x_{i-1}, x_{i+1}\right]$. We also require that the piecewise polynomials have $M-1$ continuous derivatives, when the degree of the polynomials is $2 M-1$. We show in Section 5 that once the polynomials have been constructed on one interval, a simple change of variable will give them on any interval. Therefore, our attention may be focused on obtaining a piecewise polynomial basis on one such interval. We will use $[0,2]$ for ease of notation.

Definition. A class of $M$ polynomials $\left\{P_{i}(x)\right\}$, each of degree less than or equal to $2 M-1$, is called a Hermite class of order $M$ on $[0,1]$ if, for each $i=1, \cdots, M$, the following conditions are satisfied for $j=0, \cdots, M-1$ :

$$
\begin{array}{ll}
H_{1}: & P_{i}^{(i)}(0)=0, \\
H_{2}: & P_{i}^{(i)}(1)=\delta_{i, j+1} .
\end{array}
$$

Given $\left\{P_{i}(x)\right\}$, a Hermite class of order $M$ on $[0,1]$, we define functions $\rho_{i}$ by the following:

$$
\rho_{i}(x)= \begin{cases}P_{i}(x), & x \in[0,1], \\ (-1)^{i+1} P_{i}(2-x), & x \in[1,2], \\ 0, & x \in[0,2] .\end{cases}
$$

These piecewise polynomials $\rho_{i}(x)$ clearly have support in $[0,2]$ and, by construction, they have $M-1$ continuous derivatives.

In Sections 3 and 4, we consider the problem of finding the $P_{i}(x), i=1, \cdots M$, on $[0,1]$.

3. Matrix Solution. Let $M$ be a fixed positive integer. Define $P_{i}(x)=$ $\sum_{i=0}^{M-1} C_{i j} x^{M+i}$. It is clear that $P_{i}^{(i)}(0)=0$ for $j=0, \cdots, M-1$, since $P_{i}$ has a zero of order $M$ at $x=0$. Hence, to make $\left\{P_{i}(x), i=1, \cdots, M\right\}$ into a Hermite class, it is enough to determine the $C_{i j}$ so that $H_{2}$ is satisfied. Let $f_{i}(x)=x^{M+i-1}$, for $j=$ $1, \cdots, M$, and let $A(x)$ be the matrix defined by

$$
A(x)=\left[\begin{array}{cccc}
f_{1}(x) & f_{2}(x) & \cdots & f_{M}(x) \\
f_{1}^{\prime}(x) & f_{2}^{\prime}(x) & \cdots & f_{M}^{\prime}(x) \\
\vdots & \vdots & & \\
f_{1}^{(M-1)}(x) & f_{2}^{(M-1)}(x) & \cdots & f_{M}^{(M-1)}(x)
\end{array}\right]
$$

Since the $f_{i}(x)$ are independent functions and $\operatorname{DET}(A(x))=$ Wronskian $\left[f_{1}, \cdots, f_{M}\right]$, it follows that $A=A(1)$ is invertible.

Lemma 1. Let $C=\left[C_{i j}\right]$ be the matrix of coefficients of the $P_{i}$, then $A C=I$ if and only if the $P_{i}$ are a Hermite class.

Proof. By direct computation, we have $A(x) C=\left[P_{i}^{(i-1)}(x)\right]$, and, thus, $A(1) C=$ $\left[P_{i}^{(i-1)}(1)\right]=\left[\delta_{i j}\right]=I$ if and only if $P_{1}^{(i-1)}(1)=\delta_{i i}$. Therefore, $A C=I$ if and only if the $P_{i}$ satisfy $H_{2}$. Q.E.D.

The elements of the matrix $A$ may be explicitly determined. Since $f_{i}(x)=x^{M+i-1}$, we have 


$$
\begin{aligned}
f_{i}^{(k)}(1) & =(M+j-1) \cdots(M+j-k) \\
& =(M+j-1) ! /(M+j-k-1) ! .
\end{aligned}
$$

The determinant of the matrix $A$ can probably be evaluated by using the results of Lavoie and Michaud [8], but a simple method is also available, and we will consider it instead.

Define $F_{i}(t)=f_{i}\left(e^{t}\right)$ and let $B(t)=\left[F_{i}^{(i-1)}(t)\right]$. If we also let $\lambda_{i}=M+i-1$, then since $f_{i}\left(e^{t}\right)=e^{\lambda_{i} t}$ we have

$\operatorname{DET}(B(t))=$ Wronskian $\left[e^{\lambda_{1} t}, \cdots, e^{\lambda_{M} t}\right]=e^{\left(\lambda_{1} t+\cdots+\lambda_{M} t\right)}$ Vandermonde $\left[\lambda_{1}, \cdots, \lambda_{M}\right]$. Since Vandermonde $\left[\lambda_{1}, \cdots, \lambda_{M}\right]=\prod_{1 \leqq i<i \leq m}\left(\lambda_{i}-\lambda_{i}\right)$, we obtain the following result.

Lemma 2. $\operatorname{DET}(A)= \pm \prod_{i=1}^{M}(M-i)$ !.

Proof. Since $\operatorname{DET}(A)=\operatorname{DET}(A(1))$ and $A(1)=B(0)$, we have

$$
\operatorname{DET}(A)=\text { Vandermonde }\left[\lambda_{1}, \cdots, \lambda_{M}\right]
$$

Therefore, for $\lambda_{i}=M+i-1$, we obtain

$$
\operatorname{DET}(A)= \pm \prod_{i<i}(j-i)= \pm \prod_{i=1}^{M}(M-i) ! \quad \text { Q.E.D. }
$$

The $a_{i M}$ element of $A$ is given by $a_{i M}=f_{i}^{(m-1)}(1)=\prod_{k=1}^{M-1}(M+i-k)$. Therefore, since $M+i-k \geqq i$, for $k \leqq M$, we have $a_{i M} \geqq i^{M-1}$. If we divide each row of the matrix $A$ by $a_{i M}$ and evaluate the resulting determinant, we obtain the following sequence of inequalities:

$$
\begin{aligned}
\left|\operatorname{DET}(A) / \prod_{i=1}^{M} a_{i M}\right| & \leqq\left|\operatorname{DET}(A) / \prod_{i=1}^{M} i^{M-1}\right| \\
& \leqq \prod_{i=1}^{M}(M-i) ! / \prod_{i=1}^{M} i^{M-1} \\
& \leqq \prod_{i=1}^{M}(M-i) ! /(M !)^{M-1} \\
& \ll 1 / M ! .
\end{aligned}
$$

A matrix is termed ill-conditioned whenever the absolute value of the determinant of the normalized matrix is small. The normalized matrix is obtained by dividing each row $i$ of the given matrix by $\left(\sum_{i=1}^{M} a_{i j}^{2}\right)^{1 / 2}$. The computation given above shows that $A$ is ill-conditioned because

$$
\left|\operatorname{DET}(A) / \prod_{i=1}^{M}\left(\sum a_{i i}^{2}\right)^{1 / 2}\right| \leqq\left|\operatorname{DET}(A) / \prod_{i=1}^{M} a_{i M}\right| \ll 1 / M ! .
$$

Since the matrix $A$ is ill-conditioned, another procedure for determining the $P_{i}$ is needed. In the next section, such a method is developed. Since that procedure determines the inverse of the matrix $A$, it may be applicable to general Vandermonde matrices (see Traub [13]).

Since determining each $P_{i}$ is a Hermite-Birkhoff interpolation problem of degree $2 M$, the method of Galimberti and Pereyra [4] can be applied. References [2] and [6] 
also discuss solving Vandermonde systems. In Section 5, we compare our method with that of Galimberti and Pereyra.

4. Computation of the Hermite Polynomials. In this section, we describe a recursive procedure for the computation of a Hermite class of order $M$ on $[0,1]$.

Let $q_{i}(x)=x^{M}(x-1)^{i-1} /(i-1) !, i \geqq 1$. Clearly, each $q_{i}(x)$ satisfies $H_{1}$ and, for $i \leqq M, q_{i}(x)$ is of degree $\leqq 2 M-1$. Since $q_{i+1}(x)=q_{i}(x)(x-1) / i$, we have by induction

$$
q_{i+1}^{(i)}(x)=q_{i}^{(i)}(x)(x-1) / i+(j / i) q_{i}^{(i-1)}(x) .
$$

Letting $x=1$ in this equation gives the following lemma.

LEMMA 3.

$$
q_{i+1}^{(i)}(1)=(j / i) q_{i}^{(i-1)}(1)
$$

LEMMA 4.

$$
\begin{aligned}
q_{i}^{(i)}(1) & =0 & \text { for } j<i-1, \\
& =1 & \text { for } j=i-1 .
\end{aligned}
$$

Proof. Suppose the lemma is true for $i=k$, by Lemma 3 we have

$$
q_{k+1}^{(i)}(1)=(j / k) q_{k}^{(i-1)}(1) .
$$

Thus, if $j<k$ then $q_{k}^{(j-1)}(1)=0$, and if $j=k$, then $q_{k+1}^{(i)}(1)=(k / k) q_{k}^{(k-1)}(1)=1$. The lemma is clearly true for $i=1$ and hence for all $i$. Q.E.D.

Let $P_{M}(x)=x^{M}(x-1)^{M-1} /(M-1)$ !; clearly $P_{M}$ satisfies $H_{2}$ since it has a zero of order $M-1$ at $x=1$, and $P_{M}^{(M-1)}(1)=(M-1) ! /(M-1) !=1$. Define $P_{k}(x)$ for $k=M-1, \cdots, 1$ by the following:

$$
\text { - } P_{k}(x)=q_{k}(x)+\sum_{r=k+1}^{M} C_{r k} P_{r}(x) \text {, where } C_{r k}=-q_{k}^{(r-1)}(1) \text {. }
$$

THEOREM 5. The $\left\{P_{k}(x)\right\}$ defined by $(*)$ are a Hermite class of order $M$ on $[0,1]$.

Proof. The $P_{k}$ are linear combinations of the $q_{i}$, and thus they satisfy $H_{1}$. Furthermore, since $P_{M}(x)=x^{M}(x-1)^{M-1} /(M-1)$ !, we see that $P_{M}$ satisfies $H_{2}$. For $k<M$,

$$
P_{k}(x)=q_{k}(x)+\sum_{r=k+1}^{M} C_{r k} P_{r}(x)
$$

and, in view of $q_{k}^{(j)}(1)=0$ and $P_{r}^{(j)}(1)=0$, for $j<k-1$, we see that $P_{k}^{(i)}=0$ for $j<k-1$. For $j=k-1$, only $q_{k}(1)$ remains after $j$ differentiations and Lemma 4 shows this value is 1 . Since $j>k-1$ implies $P_{r}^{(i)}(1)=1$, the definition of the $C_{r k}$ gives $P_{k}^{(i)}(1)=0$. Q.E.D.

The backward recursion formula $\left({ }^{*}\right)$ and the $C_{i j}$ are all that are needed to compute the $P_{k}$.

We have $q_{1}^{(i)}(x)=M(M-1) \cdots(M-j+1) x^{M-i}$, therefore $q_{1}^{(i)}(1)=j !\left(\begin{array}{c}M \\ i\end{array}\right)$. The following theorem supplies the values of the $C_{i j}$ in general.

THEOREM 6. If $j \geqq i$ then $q_{i}^{(i)}(1)=j !\left(\begin{array}{c}M \\ i-i+1\end{array}\right) /(i-1)$ !.

Proof. The case $i=1$ is given above. Suppose the result is true for $i=k$, then from Lemma 3 we have $q_{k+1}^{(i)}(1)=j / k q_{k}^{(i-1)}(1)$, by our induction hypothesis 
$q_{k}^{(i-1)}(1)=(j-1) !\left({ }_{j-k}^{M}\right) /(k-1)$ !. Substitution into the expression above gives $q\left(\begin{array}{l}(i) \\ k+1\end{array}\right)=j !\left({ }_{i-k}^{M}\right) / k !$. Q.E.D.

5. Computational Aspects. Section 4 gives the equations needed for computation of a Hermite class. It is quite easy to program these equations. Since the $q_{i}(x)$ can be written as binomial coefficients of powers of $x$ and the $C_{i j}$ are factorials, factorial evaluation and large integer division are the two major problems encountered. On the CDC 6600 (29S), the above procedure with

$$
E_{M}=\max _{1 \leqq i \leqq M ; 0 \leqq i \leqq M-1}\left\{\left|P_{i}^{(i)}(1)-\delta_{i, i+1}\right|,\left|P_{i}^{(i)}(0)\right|\right\},
$$

gave the following results.

\begin{tabular}{ccccccc}
\hline$M$ & 2 & 3 & 4 & 5 & 6 & 7 \\
\hline$E_{M}$ & 0 & 0 & $10^{-11}$ & $10^{-9}$ & $10^{-9}$ & $10^{-9}$ \\
\hline
\end{tabular}

For $M=4$, the $P_{i}(x)$ are

$$
\begin{aligned}
& P_{1}(x)=35 x^{4}-84 x^{5}+7.0 x^{6}-20 x^{7}, \\
& P_{2}(x)=-15 x^{4}+39 x^{5}-34 x^{6}+10 x^{7}, \\
& P_{3}(x)=2.5 x^{4}-7 x^{5}+6.5 x^{6}-2.0 x^{7}, \\
& P_{4}(x)=-1 / 6 x^{4}+.5 x^{5}-.5 x^{6}+1 / 6 x^{7} .
\end{aligned}
$$

These polynomials are exact, the error $E_{4}$ above is caused by computer roundoff in taking derivatives and evaluation.

The polynomial $P_{1}(x)$ for any $M$ is defined by $P_{1}^{(i)}(0)=0, j=0, \cdots, M-1$, $P_{1}(1)=1$, and $P_{1}^{(i)}(1)=0, j=1, \cdots, M-1$.

This polynomial is generated last by our recursive procedure, and, in all cases tried, has the most roundoff buildup. Therefore, we compared this polynomial to that generated by the Galimberti and Pereyra method [4]. We will mention the result for $M=9$, that is the 17 th degree polynomial $P_{1}(x)$. In running the program listed at the end of the paper of Galimberti and Pereyra, we encountered the following inconsistency.

Whereas part of the input to be specified for subroutine "DUALCONF" includes values of the derivatives at the various nodes, what is actually needed is the value of the $j$ th derivative divided by $j$ !. This is apparent from reading the text of the paper.

The maximum difference between the coefficients of the 17 th degree $P_{1}(x)$ polynomials occurs for the coefficient of $x^{11}$. This difference is less than $10^{-7}$.

Moreover, both coefficients are within $10^{-7}$ of the true answer. Therefore, both methods supply accurate polynomials, and both require $O\left(M^{3}\right)$ operations to compute the $M$ polynomials. It would seem that the only advantage of our method is the facility of computing all of the polynomials at the same time. This, we feel, is a minor advantage.

It should be noted that once the polynomials have been constructed on $[0,1]$, a simple change of variable is all that is needed to obtain a Hermite class defined 
for arbitrary mesh spacing. If we are given the interval $\left[h_{1}, h_{2}\right]$, let $\Delta=h_{2}-h_{1}$ and for $i=1, \cdots, M$ define

$$
R_{i}(x)=\Delta^{i-1} P_{i}\left(\left(x-h_{1}\right) / \Delta\right),
$$

where $\left\{P_{i}(x)\right\}$ is the Hermite class on $[0,1]$.

Then, for $j=0, \cdots, M-1$, we have $R_{i}^{(i)}(x)=\left(\Delta^{i-1} / \Delta^{j}\right) P_{i}^{(i)}\left(\left(x-h_{1}\right) / \Delta\right)$. Since $P_{i}^{(j)}(0)=0$, we see $R_{i}^{(i)}\left(h_{1}\right)=0$; moreover, $P_{i}^{(i)}(1)=\delta_{i, j+1}$ implies $R_{i}^{(i)}\left(h_{2}\right)=$ $\left(\Delta^{i-1} / \Delta^{i}\right) \delta_{i, i+1}$ and, therefore, $R_{i}^{(i)}\left(h_{2}\right)=\delta_{i, j+1}$. Thus, the polynomials $R_{i}(x)$ satisfy the appropriate derivative conditions at $x=h_{1}$ and $x=h_{2}$.

Now, given $\left[h_{1}, h_{2}\right] \cup\left[h_{2}, h_{3}\right]$, let $\Delta_{1}=h_{2}-h_{1}$ and $R_{i}(x)$ be defined as above, and let $\Delta_{2}=h_{3}-h_{2}$ and $S_{i}(x)$ be the equivalent polynomials for $\left[h_{2}, h_{3}\right]$.

We now define the piecewise polynomials $\rho_{i}(x), i=1, \cdots, M$, by the following formula:

$$
\rho_{i}(x)= \begin{cases}R_{i}(x), & x \in\left[h_{1}, h_{2}\right], \\ (-1)^{i+1} S_{i}\left(h_{2}+h_{3}-x\right), & x \in\left[h_{2}, h_{3}\right], \\ 0, & x \in\left[h_{1}, h_{3}\right] .\end{cases}
$$

In terms of the $P_{i}(x)$, we may write

$$
\rho_{i}(x)= \begin{cases}\Delta_{1}^{i-1} P_{i}\left(\left(x-h_{1}\right) / \Delta_{1}\right), & x \in\left[h_{1}, h_{2}\right], \\ (-1)^{i+1} \Delta_{2}^{i-1} P_{i}\left(\left(h_{3}-x\right) / \Delta_{2}\right), & x \in\left[h_{2}, h_{3}\right], \\ 0, & x \notin\left[h_{1}, h_{3}\right] .\end{cases}
$$

The $\left\{\rho_{i}(x)\right\}$ so defined are piecewise polynomials on $\left[h_{1}, h_{3}\right]$ and have $M-1$ continuous derivatives. This shows that once the $P_{i}(x)$ have been constructed on $[0,1]$, the $\rho_{i}(x)$ can be constructed for any interval of the form $\left[h_{1}, h_{2}\right] \cup\left[h_{2}, h_{3}\right]$.

One additional note, for two-dimensional rectangular meshes, is that a direct product of these polynomials supplies a basis (see [1] or [5]).

6. Applications. Given the Hermite class, the projection method discussed in [4] may be implemented. This was done for a few simple ordinary differential equations. Since integration of the polynomials times the coefficient functions and the nonhomogeneous term is required, we considered only constant coefficient problems with polynomial nonhomogeneous term.

To further simplify the calculations, the interval $[0,10]$ was used with a mesh spacing of $h=1$. The relative error was measured by computing $E=\max _{x_{i}}\left|\mu\left(x_{i}\right)-\hat{\mu}\left(x_{i}\right)\right| /\left|\mu\left(x_{i}\right)\right|$ where $\mu$ is the true solution, $\hat{\mu}$ is the Hermite approximate solution and the $x_{i}$ are one hundred equally spaced points in $[0,10]$. All problems had $\mu(0)=\mu(10)=0$ as boundary conditions. The results are summarized only for $M=2, \cdots, 7$. The case $M=7$ is polynomials of degree 13 . These are sufficient for the cases below, considering the mesh size of one.

$$
\text { 1. }-D^{2} \mu=x^{2}, \mu(x)=-x^{4} / 12+1000 x / 12
$$

\begin{tabular}{ccccccc}
\hline$M$ & 2 & 3 & 4 & 5 & 6 & 7 \\
\hline$E$ & $10^{-3}$ & $10^{-12}$ & $10^{-12}$ & $10^{-10}$ & $10^{-10}$ & $10^{-10}$ \\
\hline
\end{tabular}


2. $-D^{2} \mu+\mu=x, \mu(x)=x-\left(10 / e^{10}\right) \cdot\left(e^{x}-e^{-x}\right)$

\begin{tabular}{ccccccc}
\hline$M$ & 2 & 3 & 4 & 5 & 6 & 7 \\
\hline$E$ & $10^{-2}$ & $10^{-5}$ & $10^{-7}$ & $10^{-7}$ & $10^{-7}$ & $10^{-7}$ \\
\hline
\end{tabular}

3. $-D^{2} \mu-\mu=x, \mu(x)=-x+10 \sin x / \sin (10)$

\begin{tabular}{ccccccc}
\hline$M$ & 2 & 3 & 4 & 5 & 6 & 7 \\
\hline$E$ & $10^{-1}$ & $10^{-4}$ & $10^{-6}$ & $10^{-9}$ & $10^{-9}$ & $10^{-9}$ \\
\hline
\end{tabular}

4. $-D^{2} \mu-\mu=x^{2}, \mu(x)=-x^{2}+2+(98+2 \cos (10)) \sin x / \sin (10)-2 \cos x$

\begin{tabular}{ccccccc}
\hline$M$ & 2 & 3 & 4 & 5 & 6 & 7 \\
\hline$E$ & $10^{-1}$ & $10^{-4}$ & $10^{-6}$ & $10^{-9}$ & $10^{-9}$ & $10^{-9}$ \\
\hline
\end{tabular}

For case 1, the solution should be exact for $M \geqq 3$, the error reported is due only to roundoff. No special considerations were used in programming this problem to avoid roundoff buildup.

With this in mind, these results indicate that the use of higher order polynomials is one method of reducing approximation errors. The formulas developed in this paper allow computation of these higher polynomials quite accurately.

Acknowledgment. The authors wish to thank Professor Richard Varga and Professor Claude Poncelet for their valuable assistance in the preparation of this paper. We also wish to thank the referee for pointing out the Vandermonde references and suggesting the comparison given in Section 5 .

Bettis Atomic Power Laboratory

Westinghouse Electric Corporation

West Mifflin, Pennsylvania 15122

Department of Statistics and Computer Science

West Virginia University

Morgantown, West Virginia 26506

1. G. Birkhoff, M. H. Schultz \& R. S. VARgA, "Piecewise Hermit interpolation in one and two varables with applications to partial differential equations," Numer. Math., v. 11, 1968, pp. 232-256. MR 37 \#2404.

2. A. BJörcK \& V. Pereyra, "Solution of Vandermonde systems of equations," Math. Comp., v. 24, 1970, pp. 893-903. MR 44 \#7721.

3. H. B. Curry \& I. J. Schoenberg, "On Pólya frequency functions. IV: The fundamental spline functions and their limits," J. Analyse Math., v. 17, 1966, pp. 71-107. MR 36 \#1884.

4. G. Galimberti \& V. Pereyra, "Solving confluent Vandermonde systems," Numer. Math., v. 18, 1971 , pp. $44-60$. 
5. J. J. GoEL, "Construction of basic functions for numerical utilization of Ritz's method," Numer. Math., v.12, 1968, pp. 435-447. MR 41 \#1236.

6. S. GustaFson, "Rapid computation of interpolation formulae and mechanical quadrature rules," Comm. $A C M$, v. 14, 1971, pp. 797-801.

7. J. W. JEROME \& R. S. VARGA, "Generalizations of spline functions and applications to nonlinear boundary value and eigenvalue problems," in Theory and Applications of Spline Functions (Proc. Sem. Math. Research Center, Univ. of Wisconsin, Madison, Wis., 1968), Academic Press, New York, 1969, pp. 103-155. MR 39 \#685.

8. J. L. LAvoie \& R. Michaud, "Explicit expressions for the determinants of certain matrices," Math. Comp., v. 24, 1970, pp. 151-154. MR 41 \#1760.

9. F. R. Loscalzo, Numerical Solution of Ordinary Differential Equations by Spline Functions (SPLINDIF), Technical Summary Report \#842, Mathematics Research Center, U. S. Army, University of Wisconsin, Madison, Wis., 1968.

10. F. R. Loscalzo, On the Use of Spline Functions for the Numerical Solution of Ordinary Differential Equations, Ph.D. Thesis, University of Wisconsin, Madison, Wis., 1968; also in Technical Summary Report \#869, Mathematics Research Center, U.S. Army, University of Wisconsin, Madison, Wis., 1968.

11. F. R. Loscalzo \& I. J. Schoenberg, On the use of spline functions for the approximation of solutions of ordinary differential equations, Technical Summary Report \#723, Mathematics Research Center, U.S. Army, University of Wisconsin, Madison, Wis., 1967.

12. G. STRANG, "The finite element method and approximation theory," in Numerical Solution of Partial Differential Equations, II (SYNSPADE 1970) (Proc. Sympos., Univ. of Maryland, College Park, Md., 1970), Academic Press, New York, 1971, pp. 547-583. MR 44 \#4926.

13. J. F. Traub, "Associated polynomials and uniform method for the solution of linear problems," SIAM Rev., v.8, 1966, pp. 277-301. MR 30 \#7054.

14. R. S. VARGA, "Hermite interpolation-type Ritz methods for two-point boundary value problems," in Numerical Solution of Partial Differential Equations (Proc. Sympos. Univ. Maryland, 1965), Academic Press, New York, 1966, pp. 365-373. MR 34 \# 5302.

15. R. S. VARGA, "Accurate numerical methods for nonlinear boundary value problems," in Numerical Solution of Field Problems in Continuum Physics (Proc. Sympos. Appl. Math., Durham, N.C., 1968), SIAM-AMS Proc., vol. II, Amer. Math. Soc., Providence, R.I., 1970, pp. 152-167. MR 42 \#2650.

16. K. YosidA, Functional Analysis, Die Grundlehren der math. Wissenschaften, Band 123, Academic Press. New York; Springer-Verlag, Berlin, 1965. MR 31 \# 5054. 